\title{
Biological and Molecular Characteristics of a Novel Partitivirus Infecting the Edible Fungus Lentinula edodes
}

Mengpei Guo, Institute of Applied Mycology, Huazhong Agricultural University, Wuhan 430070, China; Yinbing Bian, Institute of Applied Mycology, Huazhong Agricultural University, Wuhan 430070, China, and Key Laboratory of Agro-Microbial Resource and Development (Ministry of Agriculture), Huazhong Agricultural University, Wuhan 430070, China; Jinjie Wang, Gangzheng Wang, and Xiaolong Ma, Institute of Applied Mycology, Huazhong Agricultural University, Wuhan 430070, China; and Zhangyi Xu, Institute of Applied Mycology, Huazhong Agricultural University, Wuhan 430070, China, and Key Laboratory of Agro-Microbial Resource and Development (Ministry of Agriculture), Huazhong Agricultural University, Wuhan 430070, China

\begin{abstract}
A new partitivirus named Lentinula edodes partitivirus 1 (LePV1) was isolated from a diseased $L$. edodes strain with severe degeneration of the mycelium and imperfect browning in bag cultures. The nucleotide sequences of LePV1 dsRNA-1 and dsRNA-2 were determined; they were 2,382 bp and 2,245 bp in length, and each contained a single ORF encoding RNA-dependent RNA polymerase ( $\mathrm{RdRp}$ ) and coat protein $(\mathrm{CP})$, respectively. The purified virus preparation contained isometric particles $34 \mathrm{~nm}$ in diameter encapsidating these dsRNAs. Phylogenetic analyses showed LePV1 to be a new member of Betapartitivirus, with

the RdRp sequence most closely related to Grapevine partitivirus. RT-PCR analysis showed that 27 of the 56 Chinese L. edodes core collection strains carry LePV1, with the virus being more common in wild strains than cultivated strains. In addition, qPCR analysis suggested that coinfection with $L$. edodes mycovirus HKB (LeV-HKB) could increase replication of the $R d R p$ gene of LePV1. This study may be essential for the development of more accurate disease diagnostics and the formulation of control strategies for viral diseases in L. edodes.
\end{abstract}

Mycovirus research was pioneered in 1962 with the report of three distinct virus-like particles (VLPs) linked to La France disease in Agaricus bisporus mushrooms (Goodin et al. 1992; Hollings 1962). A number of new fungal viruses were subsequently found, and their molecular characteristics and genomic organization were partially described (Bevan et al. 1973; Boland 1992; Jiang and Ghabrial 2004; Yu et al. 2010). The genomes of mycoviruses are made of double-stranded (ds) RNAs or single-stranded (ss) RNA or DNA; they are assembled into virus particles or exist as naked nucleic acid in fungal vesicles (Ghabrial et al. 2015).

In the majority of cases, mycoviruses with potential for biological control of plant and fungal diseases has been the subject of much research (Milgroom and Cortesi 2004; Xie and Jiang 2014). However, viral diseases of mushrooms are also important in mushroom cultivation, especially as the number of cultivated mushroom species and mushroom farms increases. Several mushroom species, including A. bisporus, Pleurotus spp., Lentinula edodes, and Flammulina velutipes, have been reported as infected with viruses (Grogan et al. 2003; Magae 2012; Magae and Sunagawa 2010; Ro et al. 2007; Tavantzis et al. 1980; Yu et al. 2003). Some of these viruses are associated with considerable morphological and physiological changes, resulting in crop losses and economic implications for the growers. For instance, two important viral diseases in A. bisporus cultivation, La France disease caused by the La France isometric virus (LIV) and/or Mushroom bacilliform virus (MBV) and "patch disease" caused by Mushroom virus $X$ (MVX), result in serious morphological deformities and are considered limiting factors in commercial $A$. bisporus cultivation, reducing both the quality and quantity of the mushrooms (Goodin et al. 1992; Rao et al. 2007; Tavantzis et al. 1980). Other symptomatic mushroom viruses such as Oyster mushroom spherical virus (OMSV), P. ostreatus spherical virus (PoSV), P. eryngii

Corresponding author: Zhangyi Xu; E-mail: zyxu2016@hotmail.com

*The $\boldsymbol{e}$-Xtra logo stands for "electronic extra" and indicates that two supplementary tables are available online.

Accepted for publication 30 December 2016.

C) 2017 The American Phytopathological Society spherical virus (PeSV), and F. velutipes browning virus (FvBV) have been associated with $P$. ostreatus die-back disease and mycelial degradation ( $\mathrm{Yu}$ et al. 2003), $P$. eryngii mycelial degradation and deformed basidiocarps (Ro et al. 2007), and brown discolored basidiocarps in F. velutipes (Magae and Sunagawa 2010), respectively.

Lentinula edodes (Berk.) Pegler, also known as Xianggu and Shiitake mushroom, is well known for its culinary uses and medicinal properties and is the second most important mushroom in terms of total world production (Bedigian 2004). Since the 1970s, many morphologically distinct VLPs or dsRNAs have been detected in L. edodes in China, South Korea, Japan, and the U.S.A. (Magae 2012; Rytter et al. 1991; Ushiyama et al. 1977; Won et al. 2013; Yao et al. 2010). The presence of viruses has been confirmed in some abnormal L. edodes strains, such as strains with mycelial degeneration, inadequate or imperfect substrate browning, and malformations of the fruiting body (Kim et al. 2013; Magae 2012; Won et al. 2013; Yao et al. 2010). However, the relationships of these mycoviruses to any harmful effects on $L$. edodes have not been determined because these mycoviruses are also found in asymptomatic strains (Kim et al. 2013). Among the viruses infecting $L$. edodes, L. edodes mycovirus $H K B$ (LeV-HKB) was the first to have its whole genome sequenced. LeV-HKB was characterized as a linear unencapsidated dsRNA element with an 11-kb-long viral genome by direct visualization of the nucleic acid using atomic force microscopy; its viral genome contains two open reading frames (ORFs) (Magae 2012). L. edodes spherical virus (LeSV), isolated from a diseased $L$. edodes, was subsequently reported to be related to $\mathrm{LeV}-\mathrm{HKB}$ because part of its genome showed very high similarity with the RdRp gene of LeV-HKB, and the N-terminal sequence of its viral coat protein was also found in the middle of the ORF1 gene of LeV-HKB (Won et al. 2013).

In this study, we report a new mycovirus, L. edodes partitivirus 1 (LePV1), which was isolated from a symptomatic L. edodes strain presenting degeneration of mycelia, abnormal browning of the bag cultures, and inability to form a fruiting body. We determined the sequence and some molecular characteristics of the two-segmented dsRNA genome and the characteristics of the LePV1 virus particles. We also investigated the occurrence of LePV1 among Chinese L. edodes core collections by RT-PCR detection. In addition, quantitative PCR (qPCR) was conducted to evaluate $R d R p$ and $C P$ gene expression in $L$. edodes infected with LePV1 and coinfected with LePV1 and LeV-HKB. 


\section{Materials and Methods}

Strains and culture conditions. L. edodes strain SX12, LMLH13, and $56 \mathrm{~L}$. edodes core collection strains (containing 21 cultivated strains and 35 wild strains) were used in this study. SX12 is a cultivated strain grown on many farms in central and eastern China, occasionally showing serious symptoms (Figs. 1A and B). In this study, two samples of SX12 were used as materials to extract dsRNA, including mycelial isolation from sawdust substrate in an abnormal cultivated bag and asymptomatic fruiting bodies. LMLH13 is a virus-free strain that presents normal mycelial performance and normal substrate browning and was used as the healthy control. The 56 strains in the Chinese $L$. edodes core collection are listed in Table 1 (Liu et al. 2015a). These 56 strains underwent LePV1 detection by RT-PCR. All of the strains were cultured in malt yeast glucose broth (MYG, containing $2 \%$ malt extract, $2 \%$ glucose, $0.1 \%$ yeast extract, and $0.1 \%$ peptone) at $150 \mathrm{rpm}$ and $25^{\circ} \mathrm{C}$ for 1 to 2 weeks (Xiang et al. 2016). Their mycelia were collected and stored at $-80^{\circ} \mathrm{C}$ for dsRNA extraction, virus particle isolation, and RT-PCR detection.

dsRNA extraction and purification. Approximately $1 \mathrm{~g}$ of frozen mycelia were ground to a fine powder in liquid nitrogen and dsRNA was obtained by phenol extraction and purified through a cellulose CF-11 column (Morris and Dodds 1979). The dsRNA was dissolved in RNase-free water and digested with DNase I and S1 Nuclease (TaKaRa, Dalian, China) to remove genomic DNA and ssRNAs. The resulting dsRNAs were electrophoresed on a $0.8 \%$ agarose gel. Two bands were obtained, excised, and purified separately using a gel extraction kit for DNA (BioTeke, Beijing, China). The quality and concentration of purified dsRNA was evaluated by electrophoresis and a DS-11 Spectrophotometer (DeNovix, Wilmington, U.S.A.).

Full-length cDNA cloning and sequencing. Purified dsRNA was used as the template for cDNA cloning. The cDNA was cloned using the method reported by Xie et al. (2011) with a minor modification. cDNA was synthesized using Moloney murine leukemia virus (M-MLV) reverse transcription (TaKaRa, Dalian, China) with the tagged random primer dN6 (5'-CGATCGATCATGATGCAATGCNNNNNN-3'). The cDNAs obtained were further purified with a silica column (BioTeke, Beijing, China) and amplified using the tagged oligonucleotide (5'-CGATCGAT CATGATGCAATGC-3') in combination with end-filling with rTaq (TaKaRa, Dalian, China). The amplification products ( $>500 \mathrm{bp}$ ) were cloned into the $p E A S Y \mathrm{~T} 1$ vector (TaKaRa, Dalian, China), and then transformed into competent Escherichia coli T1 cells. The transformants were then verified by PCR and sequenced (Tsingke, Wuhan, China). The gaps between two different clones were determined by RT-PCR using primers designed for the specific cDNA sequence obtained. The $5^{\prime}$ and $3^{\prime}$ terminal sequences of dsRNA were determined following the method of Liu et al. (2015b) with some modifications. An anchor primer PC3-T7 loop (5'GGATCCCGGGAATTCGGTAATACGACTCACTATATTTT TATAGTGAGTCGTATTA-3') was ligated to dsRNA using T4 RNA ligase (TaKaRa, Dalian, China), and then the mixture was purified with chloroform and a reverse transcription process was performed. Amplified PCR of the cDNA was implemented using the anchor-complemented primer PC2 (5'-CCGAATTCCCGGGATCC-3') and sequence-specific primers corresponding to the $5^{\prime}$ or $3^{\prime}$ terminal sequences of the dsRNA, respectively. The nucleotides were determined by sequencing three times and were then assembled. Finally, the full sequence of the cDNA was verified by RT-PCR.

Sequence analysis. Sequence similarity searches were performed using the National Center for Biotechnology Information (NCBI) databases with the blastp program (http://blast.ncbi.nlm.nih.gov/Blast. cgi). Sequence assembly and multiple-sequence alignments were carried out with DNAMAN version 8 and CLUSTAL X2, respectively. The ORF of the virus genome was deduced through the ORF Finder program of the NCBI (https://www.ncbi.nlm.nih.gov/orffinder/). Phylogenetic trees were constructed using the amino acid sequences of RdRp and CP by the neighbor-joining method using MEGA version 6.0 (Tamura et al. 2013). Amino acid sequences of other mycoviruses were used for comparative analysis and were retrieved from the NCBI database with the Batch Entrez program (http://www.ncbi. nlm.nih.gov/sites/batchentrez). The secondary structures of the terminal sequences of the dsRNAs were determined with RNAstructure version 5.8 (Reuter and Mathews 2010).
Electron microscopy and SDS-PAGE analysis of virus particles. Virus particles were purified using a sucrose density gradient centrifugation method as previously described (Liu et al. 2015b). Approximately $30 \mathrm{~g}$ of frozen mycelia were ground to a fine powder in liquid nitrogen and added to $200 \mathrm{ml}$ of $0.1 \mathrm{M}$ sodium phosphate buffer (PBS, pH 7.4) containing $0.2 \mathrm{M} \mathrm{KCl}$ and $0.5 \% \beta$-mercaptoethanol. The mixture was homogenized by shaking at $100 \mathrm{rpm}$ for $50 \mathrm{~min}$ at $10^{\circ} \mathrm{C}$ and then centrifuged at $12,000 \mathrm{rpm}$ for $20 \mathrm{~min}$ at $4^{\circ} \mathrm{C}$. The supernatant was subsequently centrifuged at $26,000 \mathrm{rpm}$ at $4^{\circ} \mathrm{C}$ for $2 \mathrm{~h}$ with an ultracentrifuge (Beckman Coulter, U.S.A.). The resulting pellets were resuspended in $0.05 \mathrm{M}$ PBS. The virus preparations were further purified using sucrose gradients ranging from 20 to $60 \%(\mathrm{w} / \mathrm{v})$ and centrifuged at $30,000 \mathrm{rpm}$ at $4{ }^{\circ} \mathrm{C}$ for $2 \mathrm{~h}$. Each fraction was individually subjected to dsRNA verification. To remove the sucrose, the gradient containing virus particles was diluted with $0.05 \mathrm{M}$ PBS and recentrifuged at $36,000 \mathrm{rpm}$ at $4{ }^{\circ} \mathrm{C}$ for $2 \mathrm{~h}$. Finally, the pellets were resuspended using $150 \mu 10.01 \mathrm{M}$ PBS and stained with $2 \%(\mathrm{w} / \mathrm{v})$ phosphor tungstic acid (PTA) for observation with a transmission electron microscope (TEM) (Hitachi, Japan). In addition, the virus particle suspension was subjected to electrophoresis analysis by $12 \%$ SDS-PAGE using the method described by Wang et al. (2014) and the gels were stained with Coomassie brilliant blue R-250 (Biosharp, Hefei, China).

RT-PCR verification of LePV1 from purified virus particles. To confirm that the virions were generated by LePV1, RNA was extracted from the purified virions with 10\% SDS and phenol/ chloroform/isoamyl alcohol (PCI, 25:24:1, v/v/v) and precipitated with ethanol. The cDNA was synthesized using M-MLV reverse transcription (TaKaRa, Dalian, China) and oligo $(\mathrm{dT})_{18}$ primer (Fan et al. 2014). RT-PCR was carried out with primers designed to amplify the full-length cDNA of LePV1 (common forward primer: GAACATCTGTCCACACGCC; dsRNA-1 reverse primer: GTTATGATTGCGAGAGGCATTC; dsRNA-2 reverse primer: AGCATTCGCTTCTTCGCAGT). The PCR reaction conditions were $95^{\circ} \mathrm{C}$ for $5 \mathrm{~min}, 30$ cycles of $95^{\circ} \mathrm{C}$ for $30 \mathrm{~s}, 55^{\circ} \mathrm{C}$ for $30 \mathrm{~s}$, $72^{\circ} \mathrm{C}$ for $1.5 \mathrm{~min}$, and $72^{\circ} \mathrm{C}$ for $10 \mathrm{~min}$. PCR products were visualized by electrophoresis on a $1 \%$ agarose gel with ethidium bromide.

RT-PCR investigation of the occurrence of LePV1 in the L. edodes core collection. Total RNA was extracted from mycelia using the STE method (Shui et al. 2008). Approximately $0.5 \mathrm{~g}$ of mycelium powder was added to $300 \mu$ l extraction buffer (RNasefree $\mathrm{H}_{2} \mathrm{O}: 10 \times$ STE: $10 \%$ SDS = 7:2:1), then $600 \mu \mathrm{l}$ PCI was added. The mixture was homogenized via vortex and centrifuged at $12,000 \mathrm{rpm}$ at $4^{\circ} \mathrm{C}$ for $10 \mathrm{~min}$. RNA was re-extracted from the supernatant with isovolumetric PCI. Then, a one-tenth volume of $3 \mathrm{M}$ sodium acetate $(\mathrm{pH} 5.2)$ and 2.5 volumes of ethanol were added to
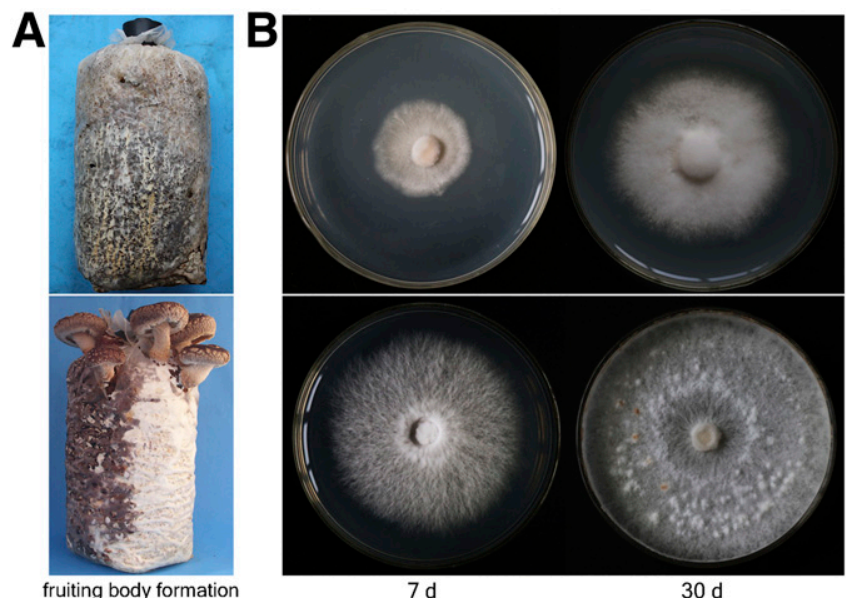

Fig. 1. Culture characteristics of a diseased strain SX12 (top) and healthy strain LMLH13 (bottom) of Lentinula edodes. A, Abnormal browning of the bag cultures and inability to form a fruiting body (top); normal browning and normal fruiting body formation (bottom). B, Degenerate mycelia (top) and normal mycelia (bottom) cultured on PDA medium for 7 days and 30 days at $25^{\circ} \mathrm{C}$ in the dark, respectively. 
precipitate the genomic RNA (Wang and Vodkin 1994). The genomic DNA in the total RNA preparation was removed using recombinant DNase I (TaKaRa, Dalian, China). The cDNA was synthesized following the protocol described by Fan et al. (2014). RT-PCR was carried out with specific primers for the RdRp gene of LePV1 (LePV1-F: 5'CGCTTTTCTTATTCTTGCCCG-3'; LePV1-R: 5'-GTTTCGAGCC CATACATAATACAG- $3^{\prime}$ ) resulting in a 315 -bp product. The $\beta$-actin gene (forward primer: 5'-GGAGAAGATTTGGCATCACACA-3'; reverse primer: $5^{\prime}$-GAAGAGCGAAACCCTCGTAGA-3') was used as positive control to confirm the successful extraction of all RNA from L. edodes. The PCR reaction conditions were $95^{\circ} \mathrm{C}$ for $5 \mathrm{~min}$ followed by 30 cycles of $95^{\circ} \mathrm{C}$ for $30 \mathrm{~s}, 55^{\circ} \mathrm{C}$ for $30 \mathrm{~s}, 72^{\circ} \mathrm{C}$ for $50 \mathrm{~s}$, and a final step of $72^{\circ} \mathrm{C}$ for $10 \mathrm{~min}$. PCR products were visualized by electrophoresis on a $1 \%$ agarose gel stained with ethidium bromide.

Quantitative analysis of the $R d R p$ and $C P$ genes of LePV1. To evaluate the $R d R p$ and $C P$ gene quantity, a quantitative PCR (qPCR) process was conducted on all $L$. edodes cDNA using AceQ qPCR SYBR Green Master Mix (Vazyme, Nanjing, China). Quantification of both gene targets was performed in triplicate. Primers for the $R d R p$ used in qPCR were RdRp-F (GTACCCTACTTACCCCGACC) and RdRp-R (CGGAACAGCCAAATTCGCAGA). Primers for the $C P$ were $\mathrm{CP}-\mathrm{F}$ (AACTTACAAGCAATCCCCACGTT) and CP-R (ACAGCACCTAGTTGAGTAGTTCC). The $\beta$-actin gene, described above, was used as the reference gene. Analysis of gene expression were carried out using CFX Manager (Bio-Rad, U.S.A.).

\section{Results}

Nucleotide sequence of LePV1 genomic dsRNAs. Two dsRNA bands were detected in SX12 both in the mycelial isolation from sawdust substrate in an abnormal cultivated bag and in asymptomatic fruiting bodies; they were confirmed to be dsRNA by DNase I and S1 nuclease digestion (Fig. 2A). Their full-length cDNA sequences were obtained and deposited in the GenBank database with accession numbers KX354971 (dsRNA-1) and KX354972 (dsRNA-2), respectively. The full-length cDNA sequence of dsRNA-1 is 2,382 bp in length and has a GC-content of $44 \%$ excluding the poly(A) tail; the dsRNA-2 is 2,245 bp in length and has a GC-content of $45 \%$. The $5^{\prime}$ - and $3^{\prime}$-untranslated regions (UTRs) are 75 and 159 bp long in dsRNA-1 and 93 and 235 bp long in dsRNA-2, respectively. Moreover, the $5^{\prime}$-UTRs and $3^{\prime}$-UTRs of the dsRNAs shared 71.28 and $42.19 \%$ similarity, respectively. They both have an adeninerich region approximately $50 \mathrm{bp}$ in length in their $3^{\prime}$ terminus (Fig. 2B). The conserved sequence of their $5^{\prime}$-UTRs was predicted to fold into a stem-loop structure (Fig. 2C); a similar stem-loop structure was also observed in RNA1 from Red clover cryptic virus 2 (RCCV-2) (Lesker et al. 2013), and was found to play an important role in dsRNA replication and virion assembly. In addition, the genome of LePV1 possesses the conserved sequence GAA at the $5^{\prime}$ terminus in both dsRNA-1 and dsRNA-2 (Fig. 2D). This $5^{\prime}$ terminal conserved GAA was also found in some fungal viruses in the genus Betapartitivirus (Fig. 2D) and the $5^{\prime}$ terminal conserved GA was found in the Alphapartitivirus and Deltapartitivirus genera (Nibert et al. 2014).

Amino acid sequence and phylogenetic analysis. Analysis of LePV1 sequences showed that dsRNA-1 contains a single long open reading frame (ORF) (from nt 76 to $n t 2223$ ) on its positive strand (Fig. 3A). The ORF encodes a 715-amino-acid (aa) protein with a predicted molecular mass of $84 \mathrm{kDa}$, containing a RdRp conserved domain (from nt 1170 to nt 1740, cd01699, 1.26e-03) belonging to

Table 1. The source of 56 strains and RT-PCR analysis of the occurrence of LePV1 in Chinese Lentinula edodes core collections ${ }^{\mathrm{a}}$

\begin{tabular}{|c|c|c|c|c|c|c|c|}
\hline \multicolumn{4}{|c|}{ Cultivated strains } & \multicolumn{4}{|c|}{ Wild strains } \\
\hline Number & Strain name & Source & $\overline{\text { LePV1 }}$ & Number & Strain name & Source & LePV1 \\
\hline 1 & S605 & Shanghai & + & 22 & EFISAAS0229 & Jingdong & - \\
\hline 2 & $\mathrm{Cr} 04$ & Sanming & - & 23 & EFISAAS0351 & Jingdong & + \\
\hline 3 & L12 & Sanming & + & 24 & 00167 & Yaoan & + \\
\hline 4 & L135 & Sanming & - & 25 & 00168 & Xianggelila & + \\
\hline 5 & L856 & Sanming & - & 26 & ACCC50786 & Huangshan & - \\
\hline 6 & S602 & Shanghai & - & 27 & GAN059 & Kang couty & + \\
\hline 7 & Guangxiang-51 & Guangdong & - & 28 & HUB039 & Shennongjia & - \\
\hline 8 & Hunong-1 & Shanghai & + & 29 & HUB040 & Shennongjia & - \\
\hline 9 & Huaxiang-8 & Wuhan & - & 30 & HUB091 & Changyang & + \\
\hline 10 & Qingke-20 & Qingyuan & + & 31 & HN002 & Chenzhou & - \\
\hline 11 & Qiu-6 & Wuhan & - & 32 & SHX044 & Liuba & - \\
\hline 12 & Rifeng-34 & Henan & - & 33 & NO.41 & Xiushui & - \\
\hline 13 & Senyuan-10 & Yichang & - & 34 & LeQc743s & Qingchuan & - \\
\hline 14 & Senyuan-1 & Yichang & - & 35 & LeWs735 & Dechang & + \\
\hline 15 & Senyuan-2 & Yichang & + & 36 & LHLy14 & Huili & + \\
\hline 16 & 430 & Wuhan & - & 37 & LHLy 217 & Huili & - \\
\hline 17 & 908 & Henan & - & 38 & LMLH14 & Mianning & - \\
\hline 18 & 868 & Zhejiang & + & 39 & LMLH36 & Mianning & + \\
\hline 19 & Xiangjiu & Guangdong & - & 40 & LMLH52 & Mianning & + \\
\hline 20 & Yuhua-2 & Biyang & - & 41 & LMLH59 & Mianning & + \\
\hline \multirow[t]{15}{*}{21} & Yuhua-4 & Biyang & - & 42 & LMLH116 & Mianning & + \\
\hline & & & & 43 & LMLHA18 & Mianning & + \\
\hline & & & & 44 & LMLHA36 & Mianning & + \\
\hline & & & & 45 & LMLHL22 & Mianning & + \\
\hline & & & & 46 & LMLHL26 & Mianning & - \\
\hline & & & & 47 & LMLHL 210 & Mianning & + \\
\hline & & & & 48 & LMYP62 & Miyi & + \\
\hline & & & & 49 & LPG82 & Puge & - \\
\hline & & & & 50 & YAASM234 & Nanhua & + \\
\hline & & & & 51 & YAASM358 & Chuxiong & + \\
\hline & & & & 52 & YAASM366 & Chuxiong & + \\
\hline & & & & 53 & YAASM1515 & Yangbi & - \\
\hline & & & & 54 & YAASM1518 & Yangbi & - \\
\hline & & & & 55 & YAASM3334 & Longling & + \\
\hline & & & & 56 & YAASM3353 & Yiliang & + \\
\hline
\end{tabular}

\footnotetext{
a + indicates LePV1 infected; - indicates no LePV1 infected.
} 
the poly(A)-type retrotransposons. Multiple comparison analysis revealed that the 715-aa protein contains six conserved motifs of RdRp (from III to VIII) (Fig. 3B). These motifs have also been described in other dsRNA viruses (Bruenn 1993; Jiang and Ghabrial 2004; Koonin 1991; Liu et al. 2015b). According to the phylogenetic tree of the amino acid sequences of RdRp, LePV1 is most closely related to the Grapevine partitivirus (AFX73019) with 59\% similarity; they are placed in a distinctive cluster of the genus Betapartitivirus (Fig. 4A). dsRNA-2 contains a single ORF (from nt 94 to nt 2010) on its positive strand (Fig. 3A). The deduced amino acid sequence includes 638-aa and encodes a protein with a predicted molecular mass of $72 \mathrm{kDa}$ that is highly homologous to CP from the family Partitiviridae. Furthermore, the phylogenetic tree constructed using the amino acid sequences of CP indicate that LePV1 is a new member of
A
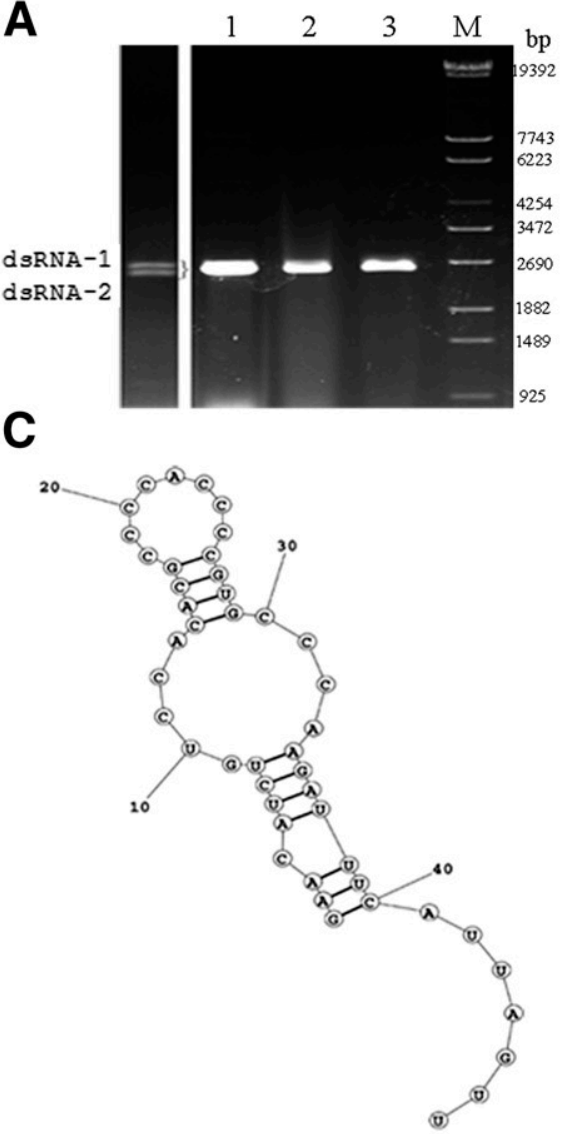

B 5'-UTR

ds RNA-1 GAACATCTGTCCACACGCCCCACCCCGTGCCCAAGAITTCAITAGTIIIAAATACTTTAA 60 ds RNA-2 GAACATCTGTCCACACGCCCCACCCCGTGCCCAAGATTTCATTAGT IG DAAATACTTTAA 60

Congengus gaacatctgtccacacgcccaccegtgcceagattcattagtt aatacttaa

ds RNA-1 ATTIDACAACTAGR............. 75

ds RNA-2 .TTMTE

Consengus ttt a aact a

3'-UTR

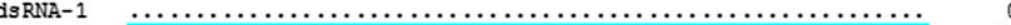
dsRNA-2 GCTGITTTITIGITITTCGATAITTACAAAGITITACGCIACCCCCCGCTCAAAAACAAT 60 Consensus

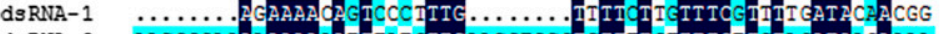

dorint-2

Consensus

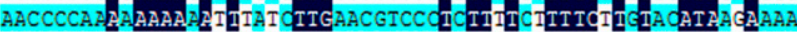

120

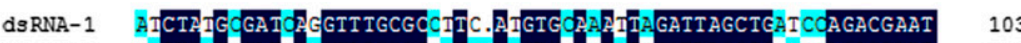
ds RNA-2 TGCTRGGIGAIIRAGTTTGCGOIOTAGA GTGIDTRCITGATTAGCTECATIAGACGAAT 180 Consensus cta g gat a gtttgcgc $\tau$ a gtg a a $t$ gattagctg agacgaat

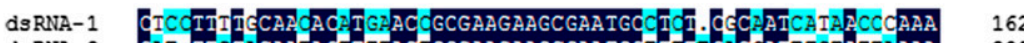

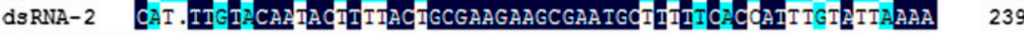
Consensus $c$ t $t$ caa ac $t$ ac gcgaagaagcgaatgc $t \tau$ c c at a aa ds RNA-1 AR CAAAAAAAR IAAAAAAAAAAAAAAAAAAAAAAAAAAAAAAAAAAAAAAAR. . 213 ds RNA-2 AR AAAAAAAAP RAAAAAAAAAAAAAAAAAAAAAAAAAAAAAAAAAAAAAARTA

Consensus aa aaaaaa aaaaaaaaaaaaaaaaaaaaaaaaaaaa

D LePV1 dsRNA-1 LePV1_dsRNA-2 HetPV $\overline{2}$ dsRNA-1 HetPV2 dsRNA-2 HetPV8_dsRNA-1 HetPV8 dsRNA-2 PoV1 dsRNA-1 PoV1_dsRNA-2 RnPV1 dsRNA-1 RnPV1_dsRNA-2 Consensus

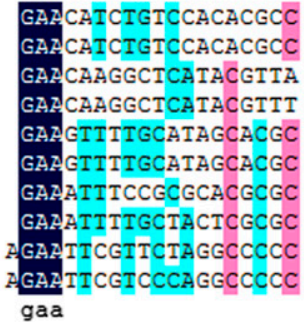

19

19

19

19

ExzsaY = -6.9

Fig. 2. Agarose gel electrophoresis analysis and molecular characteristics of the genomic dsRNA in LePV1. A, $0.8 \%$ agarose gel electrophoresis analysis of the dsRNAs extracted from mycelia of SX12. Lane 1: dsRNA without enzyme digestion; lanes 2 and 3: dsRNA digested by DNase I and nuclease S1, respectively; M: $\lambda$-EcoT14 I digest DNA marker. B, Molecular characteristics of conserved 5'-UTRs and $3^{\prime}$-UTRs (identical sequences of the $5^{\prime}$-UTR and $3^{\prime}$-UTR of the two dsRNAs are highlighted in navy blue). C, Predicted secondary structures of the conserved $5^{\prime}$-UTR sequence of LePV1. D, Molecular characteristics of the conserved sequence at the $5^{\prime}$ terminus on the plus-strand of dsRNA-1 and dsRNA-2 segments among five betapartitivirus genomes (the conserved GAA is highlighted in navy blue). The viruses used in alignment are listed in Supplementary Table S1.

A

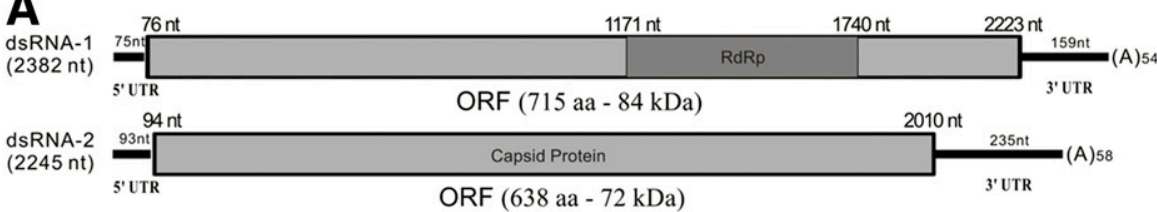

B

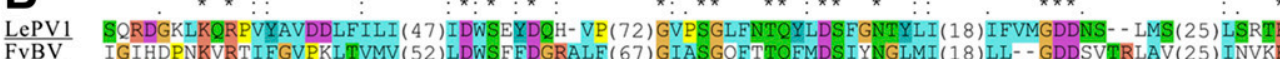

FVBV IGIHDPNKVRTIFGVPKLTVMV (52)LDWSFFDGRALF (67) GIASGQFTTQFMDSIYNGLMI (18)LL- - GDDSVTRLAV(25) INV

BCV1 VKQDDPNKMRTIWGASKPWIIA(52)LDWSRFDKRAYF (69) GIPSGLFI

GrPV

PoV1

RnPV1

RCCV2

DdV1

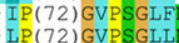

Fig. 3. Genomic organizations and conserved motifs of amino acid sequences in dsRNA-1 and dsRNA-2 from LePV1. A, Genome organization of dsRNA-1 and dsRNA-2 in LePV1. The open reading frame (ORF) and the untranslated regions (UTRs) are indicated by a gray block and a single bold line, respectively. The dark shadowing shows the conserved RdRp domain in dsRNA-1. B, Conserved motifs of amino acid sequences of RdRp (from motif III to VIII) among LePV1 and seven selected viruses in the family Partitiviridae. Detailed information of those selected viruses are described in the Supplementary Table S2. "*” indicates identical amino acid residues; ":" and "." indicate higher and lower chemically similar residues, respectively. Numbers in brackets represent the number of amino acid residues separating the motifs. 
the genus Betapartitivirus (Fig. 4B). Abbreviations of names and viral protein accession numbers for the individual viruses used in phylogenetic tree construction are listed in Supplementary Table S2.

Characterization of mycovirus particles. The virions of LePV1 consisted of spherical particles $34 \mathrm{~nm}$ in diameter (Fig. 5A) found to be concentrated in the $30 \%$ fraction. SDS-PAGE analyses of purified viral proteins from SX12 and a healthy control (strain LMLH13) were conducted. A protein band of $72 \mathrm{kDa}$ was obtained with SX12 (Fig. 5B), in accordance with our predicted molecular mass of $72 \mathrm{kDa}$ of the protein encoded by dsRNA-2. To confirm that the virions were generated by LePV1, total RNA was extracted from purified virions and RT-PCR was conducted; the results showed that products with the expected size of 2,380 bp (dsRNA-1) and 2,228 bp (dsRNA-2) were amplified from purified virions (Fig. 5C).

Occurrence of LePV1 among the $L$. edodes core collection. To investigate whether LePV1 is widespread in L. edodes, we surveyed 56 core collections of Chinese L. edodes cultivars constructed by our laboratory using ISSR and SRAP markers (Liu et al. 2015a). The results showed that many of the strains were infected with LePV1. The infection rates of cultivated strains and wild strains were $28.6 \%(6 / 21)$ and $60.0 \%$ (21/35), respectively (Fig. 6A and B). The infection rate of LePV1 among all strains in the L. edodes core collection was $48.2 \%(27 / 56)$. The infection characteristics of LePV1 are summarized in Table 1.

Coinfection with LeV-HKB. During our virus dsRNA detection of $L$. edodes strains, we found that LePV1 and LeV-HKB can infect L. edodes separately or together (Fig. 7A). In addition, the brightness of the dsRNA-1 band was nearly equivalent to the dsRNA-2 band when LePV1 infected on its own; however, the dsRNA-1 band became brighter than that of dsRNA-2 when LePV1 was coinfected with LeV-HKB, which indicates that coinfection with LeV-HKB may promote the expression of the RdRp gene in LePV1. To confirm this possibility, qPCR was conducted to detect the expression of $R d R p$ and $C P$ genes of LePV1 in the strain infected with LePV1 alone and in the strain coinfected with LePV1 and LeV-HKB, respectively. The results showed that the expression of $R d R p$ was 3.3-fold higher than $C P$ in the strain infected with LePV1 alone, while it was 18.5 -fold higher in the strain coinfected with LePV1 and LeV-HKB (Fig. 7B).

\section{Discussion}

Partitiviridae viruses have been found in two other mushroom species, i.e., P. ostreatus virus 1 (PoV1) isolated from P. ostreatus, and FvBV isolated from F. velutipes (Lim et al. 2005; Magae and
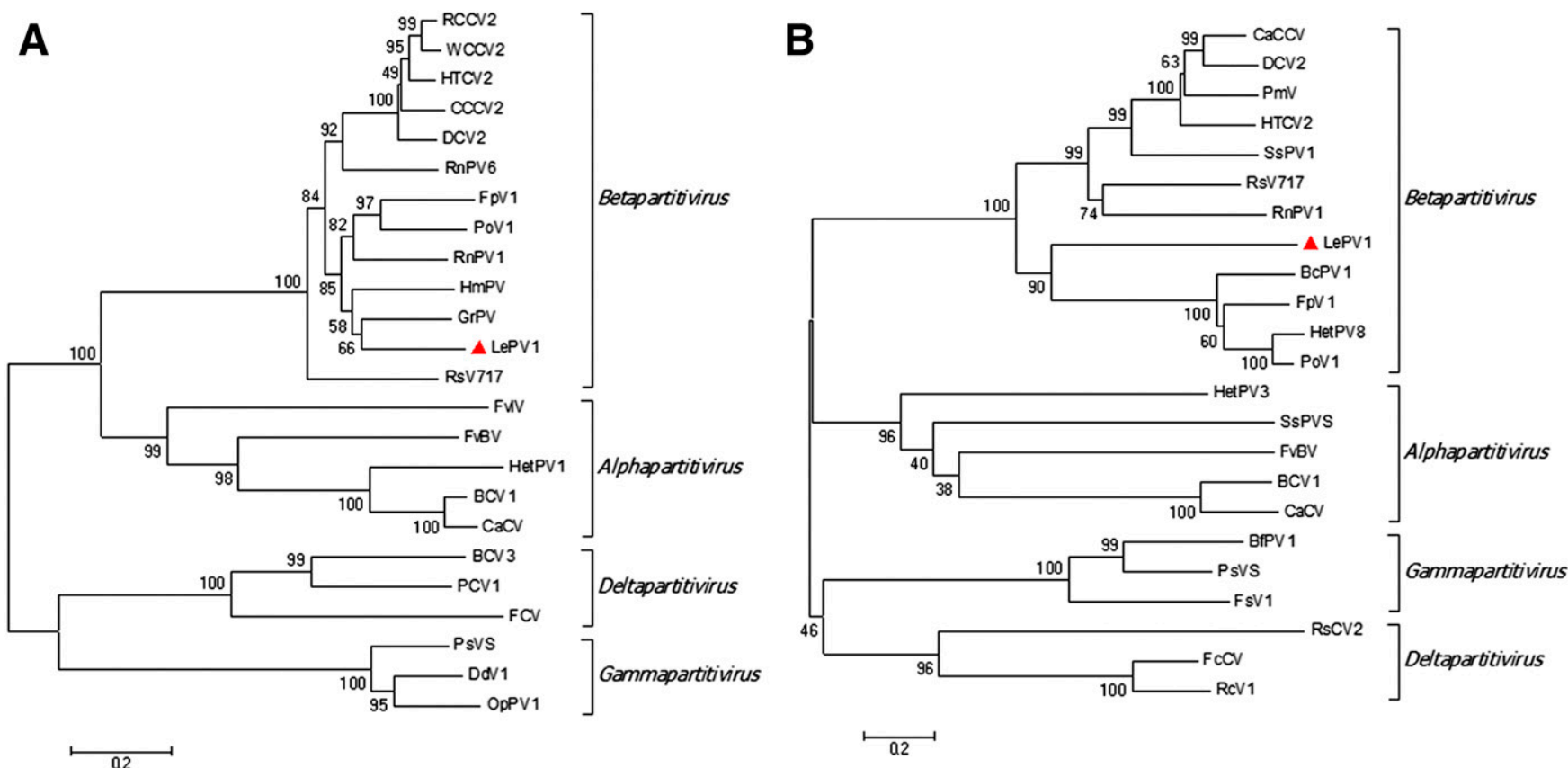

Fig. 4. Phylogenetic trees constructed based on the deduced amino acid sequences of the putative RdRp (A) and CP (B) using the neighbor-joining method (1,000 bootstrap replicates). The scale bar represents a genetic distance of 0.2 amino acid substitutions per site. The red triangle indicates the position of LePV1.
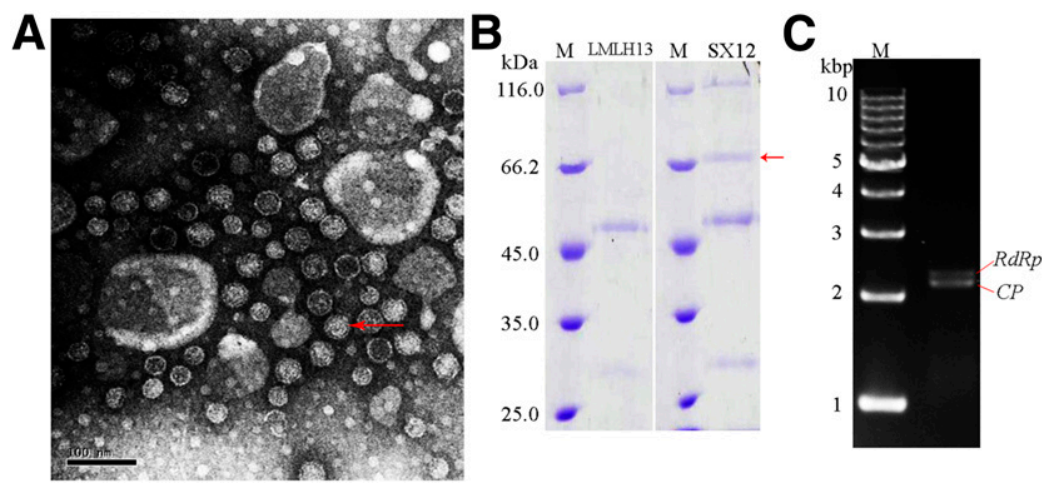

Fig. 5. Analyses of virions and capsid protein of LePV1. A, Electron micrographic characteristics of viral particles (the red arrow shows one of the viral particles). B, $12 \%$ SDS-PAGE gel electrophoresis analysis of coat protein from LePV1 virions. The red arrow shows the band (72 kDa) of capsid protein from LePV1. M: 26610 unstained protein MW marker. C, $1 \%$ agarose gel electrophoresis analysis of the RT-PCR products using the template of RNA extracted from the purified virus particles. M: $1 \mathrm{~kb}$ DNA ladder marker. 
Sunagawa 2010). In this study, we report a new partitivirus (LePV1) isolated from a diseased $L$. edodes strain SX12 (Fig. 1). The genome of LePV1 showed features typical of partitiviruses (Ghabrial et al. 2008), with two segments of approximately 2.3 and $2.2 \mathrm{kbp}$ containing a single ORF each (Figs. 2A and 3A). The larger segment encodes an RdRp, and the small one encodes the CP. As expected, they were also highly conserved, including an adenine-rich region in their $3^{\prime}$ terminus (Fig. 2B) and a stem-loop structure in the $5^{\prime}$ terminus (Fig. 2C and D). This conserved sequence and stem-loop structure of LePV1 seem to be related to RdRp recognition, virus replication and RNA packaging, and they merit further research (Lesker et al. 2013; Nibert et al. 2014). The phylogenetic trees of amino acid sequences from RdRp and CP suggest that LePV1 is a new member of the genus Betapartitivirus, within the same genus as PoV1 (Fig. 4).

Our experiments cannot directly demonstrate that LePV1 causes degeneration of mycelia and imperfect browning during cultivation of $L$. edodes SX12. However, we found that the concentration of dsRNA extracted from the mycelia of diseased strain SX12 was significantly higher than that extracted from the same weight of other strains that carry LePV1 but present normally (data not shown). In addition, we also found that the symptoms of degeneration were alleviated as the concentration of this virus dropped during the eradication of LePV1 by ribavirin (data not shown). These results indicate LePV1 is associated with the symptoms and requires further research.

The high infection rate (48.2\%) implies that LePV1 is widespread among $L$. edodes species (Fig. 6). This phenomenon was also observed for other reported L. edodes viruses such as LeSV, with a virus incidence of $66.7 \%$ among 84 commercially available cultivars (Won et al. 2013), and total of 14 unique electrophoretic dsRNA patterns in 23 out of 25 genetically diverse lines in the U.S.A. (Rytter et al. 1991). Although LePV1 was also found in some asymptomatic strains in this study, the possibility of latent infections exists since
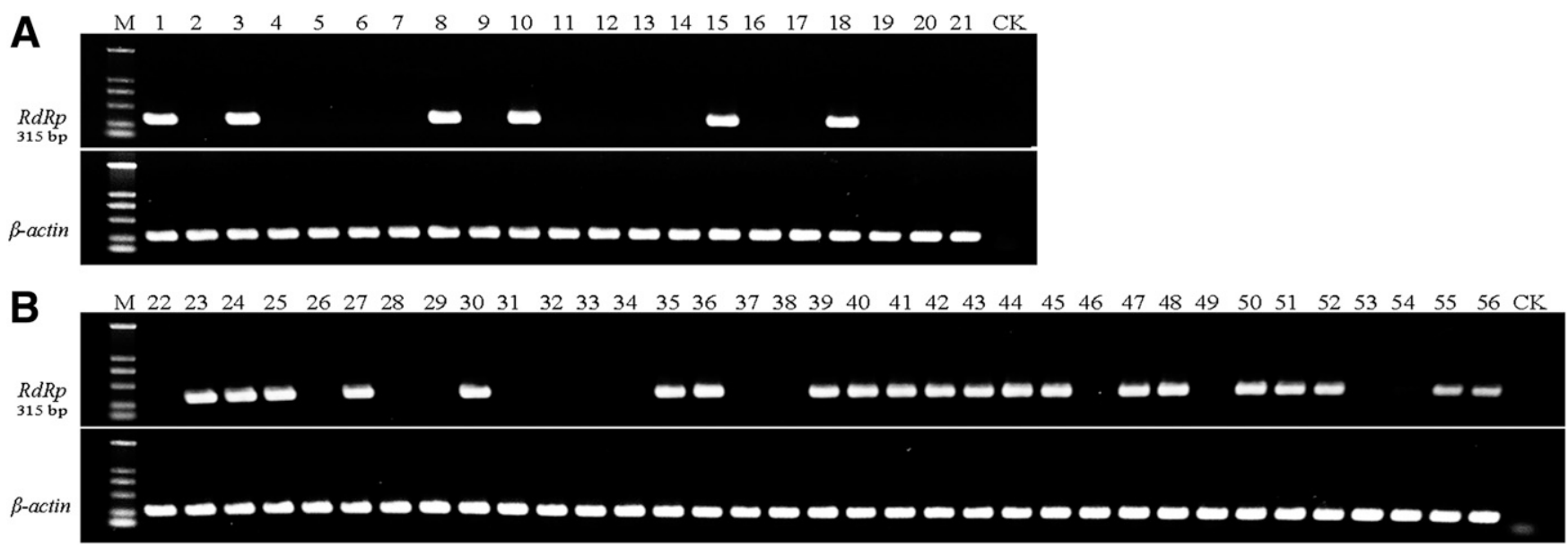

Fig. 6. RT-PCR analysis of LePV1 infection in Lentinula edodes core collections. A, Occurrence of LePV1 among 21 L. edodes cultivated strains. Numbers 1 to 21 represent 21 cultivated strains, respectively. B, Occurrence of LePV1 among 35 L. edodes wild strains. Numbers 22 to 56 represent each of the 35 wild strains. M: BM 2000 marker; CK: blank control using ddH $\mathrm{H}_{2} \mathrm{O}$ as template in the RT-PCR process. The $\beta$-actin gene was used as positive control to confirm the successful extraction of RNA.
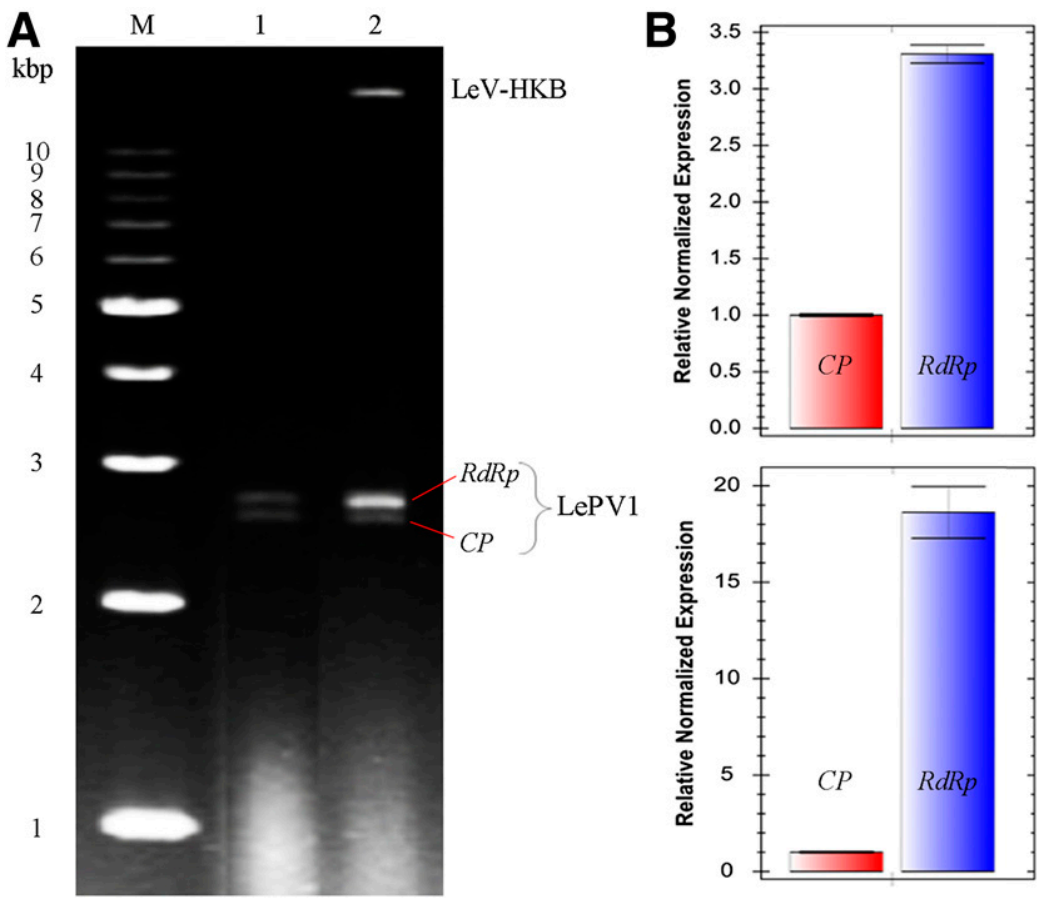

Fig. 7. Different characteristics between strain infected by LePV1 alone (lane 1 and top) and strain coinfected with LePV1 and LeV-HKB (lane 2 and bottom). A, Agarose gel electrophoresis analysis of the dsRNAs. Lane 1 and lane 2 show infection alone and coinfection, respectively. M: $1 \mathrm{~kb}$ DNA ladder marker. B, qPCR analysis of the gene expression of RdRp and CP genes. LePV1 infection alone (top), coinfection (bottom). 
members of the family Partitiviridae have been reported to have conditionally significant impacts on their hosts (Xiao et al. 2014; Zheng et al. 2014). At this point, the high frequency of virus incidence is a potential threat to the cultivation of $L$. edodes; future breeding systems for L. edodes should take care to use virus-free germplasm resources as parental materials. It has been reported that for both LIV and LeSV, wild strains have lower infection rates compared with cultivated strains (Revill and Wright 1997; Won et al. 2013); however, in this study, we found that $60.0 \%$ of the wild strains were infected with LePV1 compared with only $28.6 \%$ of the cultivated strains. This could be because most of the cultivated strains are not grouped into the core collections because of their low level of genetic diversity (a total of 89 cultivars were used as materials to construct the core collections, but only 21 cultivars were grouped into the core collections) (Liu et al. 2015a); as a result, some potentially positive strains with close kinship to the LePV1-infecting cultivars in core collections were not detected.

Coinfection of unrelated viruses are often found in plant and fungal viral diseases (Fondong et al. 2000; Harrison et al. 1997; Rao et al. 2007; Wang et al. 2014). In this study, we observed the phenomenon of coinfection with LePV1 and LeV-HKB in L. edodes (Fig. 7A) and verified a significantly higher expression of the $R d R p$ gene in LePV1 when it coinfects with LeV-HKB compared with when it infects alone (Fig. 7B). This phenomenon has also been described in several fungal and plant viruses (Karyeija et al. 2000; Sun et al. 2006; Vance 1991). For example, Cryphonectria hypovirus 1 (CHV1) coinfection with Mycoreovirus 1 (MyRV1) can elevate the replication of MyRV1, while CHV1 replication appeared to be unaffected by MyRV1 coinfection (Sun et al. 2006). This type of synergistic effect has been reported to be mediated by the papain-like cysteine protease p29 encoded by CHV1 ORF A (Sun et al. 2006). In this study, the coinfecting virus LeV-HKB contains two ORFs; its ORF1 encodes a unknown hypothetically functional protein with a NUDIX domain, and its ORF2 encodes a putative RdRp (Magae 2012). The enhancement of LePV1 replication by LeV-HKB coinfection may be mediated by the protein encoded by the LeV-HKB ORF1, which merits further research.

\section{Acknowledgments}

This study was supported by the earmarked fund for Modern Agro-industry Technology Research System on Edible Fungus of China (CARS-24), the State Key Laboratory "Key Laboratory of Integrated Pest Management on Crops in Central China, Ministry of Agriculture/Hubei Key Laboratory of Crop Diseases, Insect Pests and Weeds Control" Program (2015ZTSJJ11), and the Project of Agricultural Science and Technology Innovation Center of Hubei Province (2015-620-003001). The authors are especially grateful to Dr. Mitchell Nuhn, Clark University, U.S.A., for reviewing the manuscript.

\section{Literature Cited}

Bedigian, D. 2004. Page 310 in: Mushrooms: Cultivation, nutritional value, medicinal effects, and environmental impact, 2nd edition. P. G. Miles and S.-T. Chang, eds. CRC Press, Boca Raton, FL.

Bevan, E. A., Herring, A. J., and Mitchell, D. J. 1973. Preliminary characterization of two species of dsRNA in yeast and their relationship to the "Killer" character. Nature 245:81-86.

Boland, G. J. 1992. Hypovirulence and double-stranded RNA in Sclerotinia sclerotiorum. Can. J. Plant Pathol. 14:10-17.

Bruenn, J. A. 1993. A closely related group of RNA-dependent RNA polymerases from double-stranded RNA viruses. Nucleic Acids Res. 21:5667-5669.

Fan, X. Z., Zhou, Y., Xiao, Y., and Bian, Y. B. 2014. Cloning and characterization of two allelic glyceraldehyde-3-phosphate dehydrogenase genes in Auricularia auricula-judae. World J. Microbiol. Biotechnol. 30:181-189.

Fondong, V. N., Pita, J. S., Rey, M. E. C., de Kochko, A., Beachy, R. N., and Fauquet, C. M. 2000. Evidence of synergism between African cassava mosaic virus and a new double-recombinant geminivirus infecting cassava in Cameroon. J. Gen. Virol. 81:287-297.

Ghabrial, S. A., Castón, J. R., Jiang, D., Nibert, M. L., and Suzuki, N. 2015. 50plus years of fungal viruses. Virology 479-480:356-368.

Ghabrial, S. A., Ochoa, W. F., Baker, T. S., and Nibert, M. L. 2008. Partitiviruses: General features. Encycl. Virol. 5:68-75.

Goodin, M. M., Schlagnhaufer, B., and Romaine, C. P. 1992. Encapsidation of the La France disease-specific double-stranded RNAs in $36 \mathrm{~nm}$ isometric virus-like particles. Phytopathology 82:285-290.

Grogan, H. M., Adie, B. A. T., Gaze, R. H., Challen, M. P., and Mills, P. R. 2003. Double-stranded RNA elements associated with the MVX disease of Agaricus bisporus. Mycol. Res. 107:147-154.
Harrison, B. D., Zhou, X., Otim-Nape, G. W., Liu, Y., and Robinson, D. J. 1997. Role of a novel type of double infection in the geminivirus-induced epidemic of severe cassava mosaic in Uganda. Ann. Appl. Biol. 131:437-448.

Hollings, M. 1962. Viruses associated with a die-back disease of cultivated mushroom. Nature 196:962-965.

Jiang, D., and Ghabrial, S. A. 2004. Molecular characterization of Penicillium chrysogenum virus: Reconsideration of the taxonomy of the genus Chrysovirus. J. Gen. Virol. 85:2111-2121.

Karyeija, R. F., Kreuze, J. F., Gibson, R. W., and Valkonen, J. P. T. 2000 Synergistic interactions of a potyvirus and a phloem-limited crinivirus in sweet potato plants. Virology 269:26-36.

Kim, J., Yun, S., Park, S., Ko, H., and Kim, D. 2013. Occurrence of dsRNA mycovirus (LeV-FMRI0339) in the edible mushroom Lentinula edodes and meiotic stability of LeV-FMRI0339 among monokaryotic progeny. Plant Pathol. J. 29:460-464.

Koonin, E. V. 1991. The phylogeny of RNA-dependent RNA polymerases of positive-strand RNA viruses. J. Gen. Virol. 72:2197-2206.

Lesker, T., Rabenstein, F., and Maiss, E. 2013. Molecular characterization of five betacryptoviruses infecting four clover species and dill. Arch. Virol. 158: 1943-1952.

Lim, W., Jeong, J. H., Jeong, R.-D., Yoo, Y. B., Yie, S. W., and Kim, K.-H. 2005 Complete nucleotide sequence and genome organization of a dsRNA partitivirus infecting Pleurotus ostreatus. Virus Res. 108:111-119.

Liu, J., Wang, Z. R., Li, C., Bian, Y. B., and Xiao, Y. 2015a. Evaluating genetic diversity and constructing core collections of Chinese Lentinula edodes cultivars using ISSR and SRAP markers. J. Basic Microbiol. 55:749-760.

Liu, L., Wang, Q., Cheng, J., Fu, Y., Jiang, D., and Xie, J. 2015b. Molecular characterization of a bipartite double-stranded RNA virus and its satellite-like RNA co-infecting the phytopathogenic fungus Sclerotinia sclerotiorum. Front. Microbiol. 6:406.

Magae, Y. 2012. Molecular characterization of a novel mycovirus in the cultivated mushroom, Lentinula edodes. Virol. J. 9:60.

Magae, Y., and Sunagawa, M. 2010. Characterization of a mycovirus associated with the brown discoloration of edible mushroom, Flammulina velutipes. Virol. J. 7:342.

Milgroom, M. G., and Cortesi, P. 2004. Biological control of chestnut blight with hypovirulence: A critical analysis. Annu. Rev. Phytopathol. 42:311-338.

Morris, T. J., and Dodds, J. A. 1979. Isolation and analysis of double-stranded RNA from virus-infected plant and fungal tissue. Phytopathology 69:854-858.

Nibert, M. L., Ghabrial, S. A., Maiss, E., Lesker, T., Vainio, E. J., Jiang, D., and Suzuki, N. 2014. Taxonomic reorganization of family Partitiviridae and other recent progress in partitivirus research. Virus Res. 188:128-141.

Rao, J. R., Nelson, D. W. A., and McClean, S. 2007. The enigma of doublestranded RNA (dsRNA) associated with mushroom virus X (MVX). Curr. Issues Mol. Biol. 9:103-21.

Reuter, J. S., and Mathews, D. H. 2010. RNAstructure: software for RNA secondary structure prediction and analysis. BMC Bioinformatics 11:129.

Revill, P. A., and Wright, P. J. 1997. RT-PCR detection of dsRNAs associated with La France disease of the cultivated mushroom Agaricus bisporus (Lange). Imbach. J. Virol. Methods 63:17-26.

Ro, H., Kang, E., Yu, J., Lee, T.-S., Lee, C.-W., and Lee, H. 2007. Isolation and characterization of a novel mycovirus, PeSV, in Pleurotus eryngii and the development of a diagnostic system for it. Biotechnol. Lett. 29:129-135.

Rytter, J. L., Royse, D. J., and Romaine, C. P. 1991. Incidence and diversity of double-stranded RNA in Lentinula edodes. Mycologia 83:506-510.

Shui, P., Zheng, X., Lin, J., and Guo, L. 2008. A simple and high-quality method of total RNA extraction from edible mushroom. (in Chinese) Acta Edulis Fungi 15:32-36.

Sun, L., Nuss, D. L., and Suzuki, N. 2006. Synergism between a mycoreovirus and a hypovirus mediated by the papain-like protease p29 of the prototypic hypovirus CHV1-EP713. J. Gen. Virol. 87:3703-3714.

Tamura, K., Stecher, G., Peterson, D., Filipski, A., and Kumar, S. 2013. MEGA6: Molecular evolutionary genetics analysis version 6.0. Mol. Biol. Evol. 30: 2725-2729.

Tavantzis, S. M., Romaine, C. P., and Smith, S. H. 1980. Purification and partial characterization of a bacilliform virus from Agaricus bisporus: A singlestranded RNA mycovirus. Virology 105:94-102.

Ushiyama, R., Nakai, Y., and Ikegami, M. 1977. Evidence for double-stranded RNA from polyhedral virus-like particles in Lentinus edodes (Berk.). Sing. Virology 77:880-883.

Vance, V. B. 1991. Replication of potato virus X RNA is altered in coinfections with potato virus Y. Virology 182:486-494.

Wang, C., and Vodkin, L. O. 1994. Extraction of RNA from tissues containing high levels of procyanidins that bind RNA. Plant Mol. Biol. Report. 12:132-145.

Wang, L., Jiang, J., Wang, Y., Hong, N., Zhang, F., Xu, W., and Wang, G. 2014 Hypovirulence of the phytopathogenic fungus Botryosphaeria dothidea: association with a coinfecting chrysovirus and a partitivirus. J. Virol. 88: 7517-7527.

Won, H., Park, S., Kim, D.-K., Shin, M. J., Kim, N., Lee, S.-H., Kwon, Y., Ko, H. K., Ro, H., and Lee, H. 2013. Isolation and characterization of a mycovirus in Lentinula edodes. J. Microbiol. 51:118-122.

Xiang, X., Li, C., Li, L., Bian, Y., Kwan, H. S., Nong, W., Cheung, M. K., and Xiao, Y. 2016. Genetic diversity and population structure of Chinese Lentinula edodes revealed by InDel and SSR markers. Mycol. Prog. 15:37. 
Xiao, X., Cheng, J., Tang, J., Fu, Y., Jiang, D., Baker, T. S., Ghabrial, S. A., and Xie, J. 2014. A novel partitivirus that confers hypovirulence on plant pathogenic fungi. J. Virol. 88:10120-10133.

Xie, J., and Jiang, D. 2014. New insights into mycoviruses and exploration for the biological control of crop fungal diseases. Annu. Rev. Phytopathol. 52:45-68.

Xie, J., Xiao, X., Fu, Y., Liu, H., Cheng, J., Ghabrial, S. A., Li, G., and Jiang, D. 2011. A novel mycovirus closely related to hypoviruses that infects the plant pathogenic fungus Sclerotinia sclerotiorum. Virology 418:49-56.

Yao, L., Chen, C., Zhang, Z., Ying, G., Sun, X., Lv, M., Xue, Z., and Li, L. 2010. The partial genome cDNA cequence of a novel dsRNA virus from Lentinus edodes and the virus detected by RT-PCR. (in Chinese) Microbiol. China 37: 61-70.

Yu, H. J., Lim, D., and Lee, H.-S. 2003. Characterization of a novel single-stranded RNA mycovirus in Pleurotus ostreatus. Virology 314:9-15.

Yu, X., Li, B., Fu, Y., Jiang, D., Ghabrial, S. A., Li, G., Peng, Y., Xie, J., Cheng, J., Huang, J., and Yi, X. 2010. A geminivirus-related DNA mycovirus that confers hypovirulence to a plant pathogenic fungus. Proc. Natl. Acad. Sci. 107:8387-8392.

Zheng, L., Zhang, M., Chen, Q., Zhu, M., and Zhou, E. 2014. A novel mycovirus closely related to viruses in the genus Alphapartitivirus confers hypovirulence in the phytopathogenic fungus Rhizoctonia solani. Virology 456-457:220-226. 